\title{
Template-Induced Growth of Close-Packed and Non-Close-Packed Colloidal Crystals during Solvent Evaporation
}

\author{
J. P. Hoogenboom, ${ }^{\star}, t, \neq, \S$ C. Rétif, ${ }^{\dagger}$ E. de Bres, ${ }^{\dagger}$ M. van de Boer, ${ }^{\dagger}$ \\ A. K. van Langen-Suurling," J. Romijn," and A. van Blaaderen ${ }^{*, t, \neq}$
}

F.O.M. Institute for Atomic and Molecular Physics, Kruislaan 407, 1098 SJ Amsterdam, The Netherlands, Soft Condensed Matter, Debye Institute, Utrecht University, Princetonplein 5, 3584 CC Utrecht, The Netherlands, and Delft Institute of Microelectronics and Submicron Technology, 2600 GB Delft, The Netherlands

Received October 6, 2003

\begin{abstract}
Template-induced colloidal deposition during solvent evaporation is a promising technique for extending the possibilities of nanosphere lithography and the creation of photonic band gap materials. We investigated the influence of the parameters that determine the surface topography of templates on colloidal crystal structure. On pillar-shaped templates, large defect-free square symmetric monolayers, ordered vacancy arrays, and body-centered cubic (bcc) and simple cubic (sc) colloidal crystals could be grown. Close-packed crystals displayed defects and large defect grains. Our results indicate that this may be avoided when the direction of gravity with respect to the substrate is changed.
\end{abstract}

The ability of colloids to self-assemble into 2D and 3D crystalline structures lies at the heart of many studies in nanoand micrometer-scale materials science. ${ }^{1}$ Especially in the field of photonic band gap materials, colloidal crystals are being routinely used. ${ }^{2}$ Furthermore, 2D and thin 3D colloidal crystals can be used as a mask for creating regular arrays of nanometer-sized features with a technique called nanosphere lithography. ${ }^{3-5}$ One of the simplest and most frequently used techniques for assembling 2D and 3D colloidal crystals is colloidal crystallization during solvent evaporation. ${ }^{6-9}$

Apart from its simplicity and low cost, the main advantages of the technique are (i) the possibility to grow millimeter-sized single crystals with a hexagonal (111) orientation of the top surface and (ii) the possibility to control the thickness of the deposited crystal by varying the initial particle volume fraction. ${ }^{7}$ A major limitation is that the crystal symmetry and 3D orientation of the crystal cannot be influenced. The use of a patterned substrate, or template, provides the possibility to direct colloidal crystallization

\footnotetext{
* Corresponding authors. E-mail: j.p.hoogenboom@ utwente.nl, a.vanblaaderen@phys.uu.nl.

F. O. M. Institute for Atomic and Molecular Physics.

$\doteqdot$ Utrecht University.

$\S$ Present address: Applied Optics Group, MESA ${ }^{+}$Research Institute, University of Twente, P.O. Box 217, 7500 AE Enschede, The Netherlands.

"Delft Institute of Microelectronics and Submicron Technology.
}

epitaxially. ${ }^{10}$ Recent experiments on colloidal epitaxy under equilibrium conditions have shown the growth of crystal structures that are metastable in bulk crystallization and thus would not grow without the use of the template. ${ }^{11,12}$ Several papers have reported results on template-induced colloidal assembly during solvent evaporation, but the systems consisted of (repeated ${ }^{13}$ ) 2D deposits of micrometer-scale particles ${ }^{14}$ or showed the frequent occurrence of defects and a lack of long-range order. ${ }^{15}$

In this letter, we demonstrate the templated growth of 100nm-radius particles in colloidal crystals with close-packed and non-close-packed symmetry. The crystals were grown by convective assembly on a substrate placed in a vertical setup (i.e., parallel to gravity). We will address the influence of various parameters that determine the template topography on 2D and 3D colloidal crystal structure. Our results indicate that the direction of the gravitational field plays a crucial role in template-directed convective assembly.

Silica particles with diameters of $d=202$ and $220 \mathrm{~nm}$ (polydispersity $\sigma \approx 0.005$ ) were deposited onto a substrate that was vertically placed in a slowly evaporating dispersion in ethanol, as described by Jiang et al. ${ }^{7}$ Particles with a diameter of $870 \mathrm{~nm}(\sigma \approx 0.035)$ were deposited following the same procedure in an oven at a temperature of $T=65.5$ $\pm 0.5^{\circ} \mathrm{C} .{ }^{9,14,16} \mathrm{We}$ used several different substrate materials 


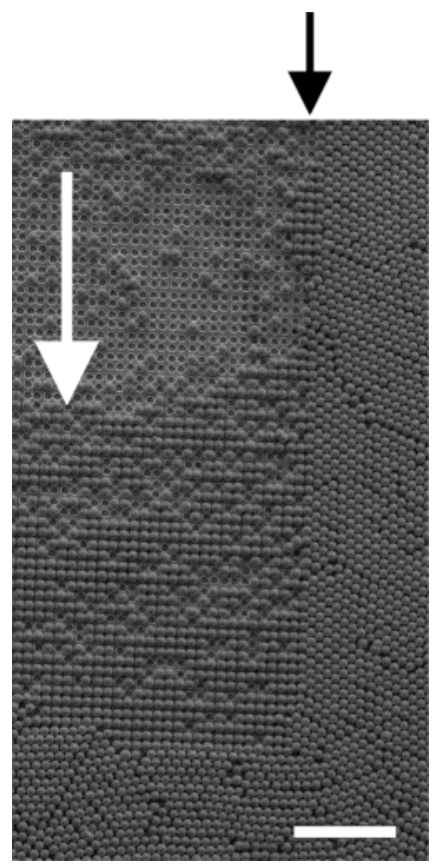

Figure 1. SEM image of 870-nm-diameter silica colloids arranged on a square-symmetric (fcc(100)) all-silicon template (depth $\approx 350$ $\mathrm{nm}$ ) after vertical controlled drying. The white arrow indicates the receding direction of the liquid-air interface during evaporation. The black arrow denotes the edge of the templated area. The scale bar denotes $10 \mu \mathrm{m}$.

including silicon, glass, and polyurethane (Norland OA 71 and 73) -coated glass. Results were always best using silicon substrates. Therefore, the results presented below concern silicon substrates unless stated otherwise.

The silicon substrates were partially patterned using a combination of electron-beam lithography and reactive ion etching. The details of these procedures have been described elsewhere. ${ }^{17}$ Patterns with a square-symmetric face-centeredcubic (100) (fcc(100)) arrangement of holes were made with lattice spacings of $a=175,200,205$, and $225 \mathrm{~nm}$ for the smaller sphere sizes and $a=850$ and $900 \mathrm{~nm}$ for the larger spheres. Patterns with a fcc(110) arrangement were made with lattice spacings of 205, 210, and $215 \mathrm{~nm}$. The spacing was found to be reproduced to $\pm 5 \mathrm{~nm}$ as measured with scanning electron microscopy (SEM). The depth of the pattern was initially set at $a / 2$ but was later varied as will be described below. Patterns always consisted of at least 500 $\times 500$ unit cells.

Figure 1 shows the result of the deposition of $870-\mathrm{nm}$ diameter particles from a $0.3 \%$ volume fraction suspension. The deposited crystal displayed clear monolayer steps upon visual inspection. All areas with a coverage of more than a single layer showed a hexagonal arrangement of particles in the top layer, both on the templated and the flat areas. Only for submonolayer coverage did the particles follow the template pattern, as shown in the left part of Figure 1. As can be seen in Figure 1, the single-layer symmetry reconstructs to hexagonal when particle coverage approaches a complete single layer. Note that the orientation of the hexagonal layer on the template differs from that on the untemplated silicon (right-hand side of the black arrow in
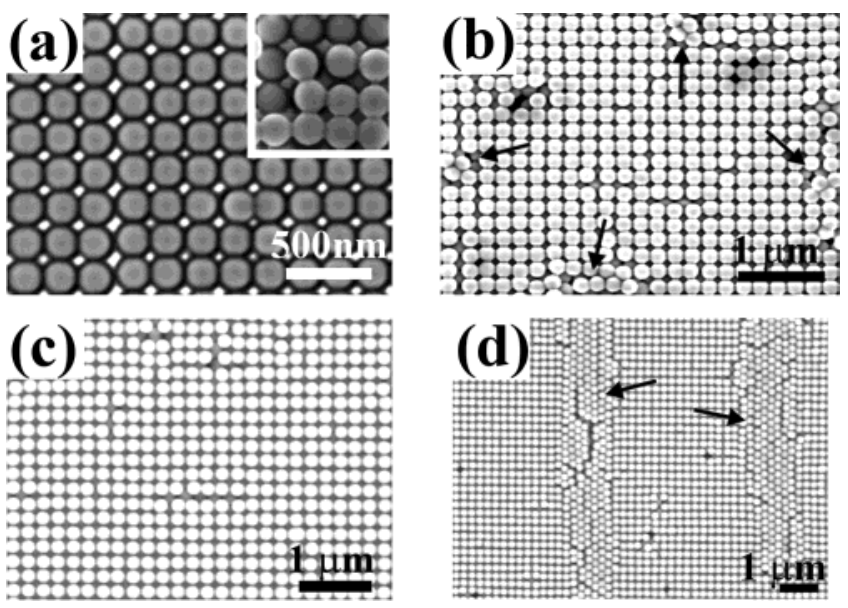

Figure 2. SEM images of colloidal particles arranged on a squaresymmetric pillar-shaped silicon template. The thickness of the crystal is (a) one layer, (b) two layers, (c) three layers, and (d) four layers. The template is visible in part a as bright spots between the particles. Arrows in part $b$ mark small defects resulting from in-plane close packing. Arrows in part $d$ indicate two large hexagonally close-packed defect grains. The inset in part a shows an incomplete top layer on a template with a depth of $\sim 300 \mathrm{~nm}$.

Figure 1) because of the presence of the template. Furthermore, the presence of the template leads to an increase in defects and dislocations in the reconstructed hexagonal layer compared to the number in the monolayer deposited on the flat silicon area.

The reason for the occurrence of the hexagonal surface reconstruction might be twofold. First, it could result from a mismatch between the template lattice spacing and colloidal dimensions. Instead of forming a defective crystal structure, as observed in equilibrium colloidal epitaxy experiments, the presence of a convective flow field and strong capillary interactions between the particles might favor the possibly less defective reconstructed structure. Second, the reconstruction could be an intrinsic effect of the growth process during solvent evaporation. In that case, the compacting flow field and possibly the capillary interactions in the last stage of the drying process favor the close-packed structure with the surface-area-minimized hexagonal top surface.

We investigated the influence of template parameters further using the smaller particles and lattice constants described above, reducing the mismatch to within the experimental accuracy of determining particle sizes and template spacing. The thickness of the deposited colloidal film was tuned by a variation of the volume fraction. ${ }^{7}$ Furthermore, the degree of support for a layer with a symmetry and/or spacing other than that of the template (such as a hexagonal layer) was varied by a variation of the hole radius $(R)$. We observed the ordering of a square-symmetric colloidal monolayer only on pillar-shaped templates (i.e., $2 R$ $\geq a$ ), as in Figure 2a. For all templates and for both particle sizes, we always observed a hexagonal reconstruction if $2 R$ $<a$.

For multilayer deposits on the pillar-shaped template, defects occur in the top layer starting with a two-layer deposit shown in Figure 2b. For deposits of more than two layers, crystalline regions with a fcc(100)-oriented top layer could 


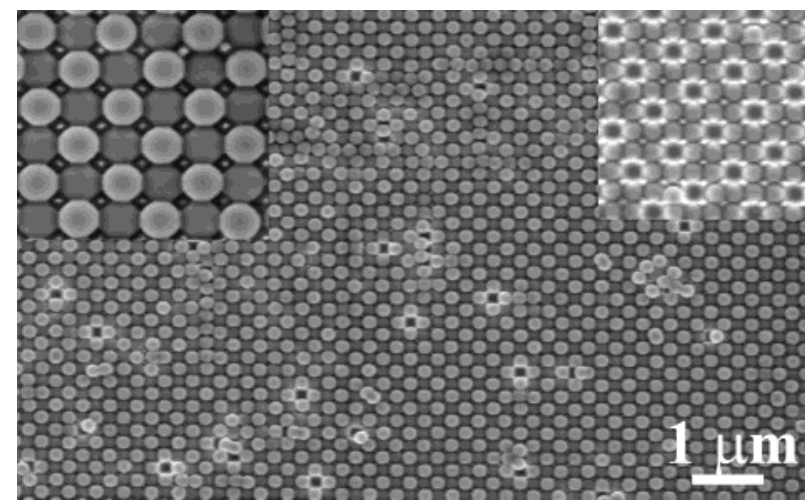

Figure 3. SEM image of a two-layer BCC structure on a template with a pitch $a=200 \mathrm{~nm}$, particle diameter $d=202 \mathrm{~nm}$, and volume fraction $\varphi=4 \times 10^{-4}$. The left inset shows an enlargement of the BCC structure; the right inset shows the ordering of vacancies in a monolayer deposited at lower particle concentration.

be found (Figure 2c), but the overall crystal symmetry on the templated area was broken by large, reconstructed (111)oriented crystal grains (Figure 2d). In general, the crystal grains with a (111) top surface were found to be larger with increasing crystal thickness, but we did not observe any systematic dependence. In fact, for deposits thicker than four layers, complete coverage of the templated area with a crystal with a hexagonal top layer was found to occur more often than a polycrystalline "broken" structure. We did not observe any epitaxial effect when depositing particles on templates with fcc(110) symmetry with lattice spacings of 205, 210, and $215 \mathrm{~nm}$ for 3- to 5-layer deposits.

These results were not found to depend on the depth of the pattern. Interestingly, we observed the ordering of a submonolayer of particles (as in the top left of Figure 1) on the template positions on a template with a depth as small as $d / 10$. Furthermore, an increase in the depth in the fcc(100) template consisting of pillars provides a means to grow a simple cubic (SC) crystal (inset of Figure 2a). When the depth exceeds $3 d / 2$, the particles are ordered on top of each other between the pillars. The use of polystyrene particles and the removal of the template by etching with HF (or vice versa, the use of a polymeric template with silica particles in combination with the pyrolysis of the polymer) results in a crystal with a volume fraction as low as 0.52 . Colloidal crystals with a volume fraction below close packing have so far been made only using a layer-by-layer procedure with a binary system. ${ }^{13}$

On the fcc(100) pillar template with a pitch comparable to the particle diameter, colloids arrange in an in-plane nonclose-packed structure. In a single layer, every particle is surrounded by four vacancies (Figure 3). Clearly, this inplane structure provides a unique template for further growth, which gives the possibility for 3D non-close-packed crystal growth. The resulting 3D structure is that of a body-centered cubic (bcc) crystal, with a volume fraction of 0.68 . Interestingly, we also observed symmetrical arrangements of vacancies over distances as large as four particle diameters (along the template axes), as shown in the right inset in Figure 3. This again indicates the importance of surface area minimization during evaporative self-assembly. ${ }^{13}$

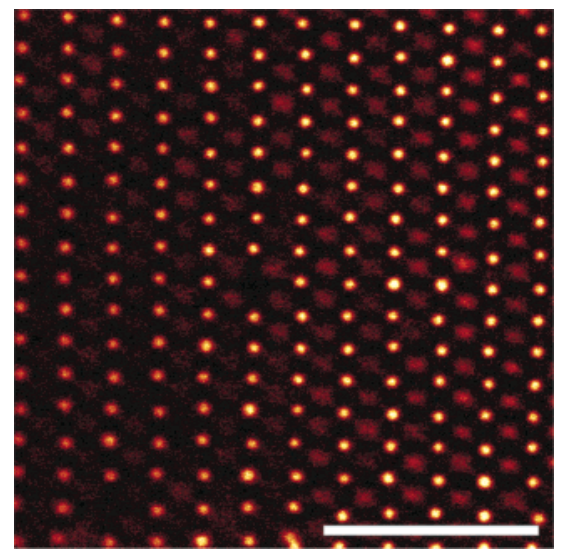

Figure 4. Confocal microscopy image of the first layer of fluorescent core - shell silica particles $(d=1.4 \mu \mathrm{m})$ arranged on a fcc(110) template in a tilted-horizontal setup. Particles in the second layer are vaguely visible as well. The scale bar indicates $10 \mu \mathrm{m}$.

Finally, we want to suggest a procedure to avoid the defective hexagonal arrangement of particles in templateinduced convective self-assembly described above. To have gravity act as an additional force directed toward the template, the template was tilted so that the angle between the surface normal and gravity was $2-10^{\circ}$. In this way, two layers with fcc(110) symmetry were grown on a polyurethane template, as shown in Figure 4. In principle, conditions can be chosen such that particles completely sediment before drying, thus crystallizing on the template under equilibrium conditions. In this respect, it is interesting that we have observed similar strain effects in crystals grown in a tilted horizontal setup on a mismatched template as in equilibrium colloidal epitaxy. ${ }^{11}$ Recently, Yin and co-workers have furthermore demonstrated the growth of fcc(100)-oriented colloidal crystals using an alternative templating procedure. ${ }^{18}$ In their setup, ${ }^{19,20}$ the patterned substrate is similarly oriented with respect to gravity as in our tilted horizontal setup.

In conclusion, we have presented results on templateinduced colloidal crystallization during solvent evaporation with the templated substrate placed parallel to gravity. On a template consisting of pillars arranged in a square symmetry, a large, defect-free, square-symmetric close-packed 2D crystal was grown. At submonolayer coverage, we observed colloidal arrays with an ordered distribution of vacancies. Furthermore, 3D non-close-packed simple cubic and bodycentered cubic crystals could be grown. However, when growing large close-packed crystals in any orientation other than with hexagonal close-packed planes parallel to the substrate, defects and large, hexagonally arranged crystal grains occurred. Close-packed crystals could be oriented when the template was placed with the surface normal at an angle of 2 to $10^{\circ}$ with respect to gravity. These results indicate that gravity might play a crucial role in the convective self-assembly process.

Acknowledgment. We thank Krassimir Velikov for many helpful discussions and Herman Offerhaus for a critical reading of the manuscript. This work is part of the research program of the Stichting voor Fundamenteel Onderzoek der 
Materie (FOM), which is financially supported by the Nederlandse Organisatie voor Wetenschappelijk Onderzoek.

\section{References}

(1) Grier, D. G. MRS Bull. 1998, 23, 21-21.

(2) Imhof, A. In Nanoscale Materials; Liz-Marzan, L. M., Kamat, P. V., Eds.; Kluwer Academic: Boston, MA, 2003; pp 423-454.

(3) Deckman, H. W.; Dunsmuir, J. H. Appl. Phys. Lett. 1982, 41, 377379.

(4) Haynes, C. L.; Van Duyne, R. P. J. Phys. Chem. B 2001, 105, 55995611.

(5) Burmeister, F.; Schafle, C.; Keilhofer, B.; Bechinger, C.; Boneberg, J.; Leiderer, P. Adv. Mater. 1998, 10, 495-497.

(6) Denkov, N. D.; Velev, O. D.; Kralchevsky, P. A.; Ivanov, I. B.; Yoshimura, H.; Nagayama, K. Nature 1993, 361, 26-26.

(7) Jiang, P.; Bertone, J. F.; Hwang, K. S.; Colvin, V. L. Chem. Mater. 1999, 11, 2132-2140.

(8) Gu, Z. Z.; Fujishima, A.; Sato, O. Chem. Mater. 2002, 14, 760765.

(9) Vlasov, Y. A.; Bo, X. Z.; Sturm, J. C.; Norris, D. J. Nature 2001, 414, 289-293.
(10) van Blaaderen, A.; Ruel, R.; Wiltzius, P. Nature 1997, 385, 321324.

(11) Hoogenboom, J. P.; van Langen-Suurling, A. K.; Romijn, J.; van Blaaderen, A. Phys. Rev. Lett. 2003, 90, 138301.

(12) Hoogenboom, J. P.; Yethiraj, A.; van Langen-Suurling, A. K.; Romijn, J.; van Blaaderen, A. Phys. Rev. Lett. 2002, 89, 256104.

(13) Velikov, K. P.; Christova, C. G.; Dullens, R. P. A.; van Blaaderen, A. Science 2002, 296, 106-109.

(14) Ye, Y. H.; Badilescu, S.; Truong, V. V.; Rochon, P.; Natansohn, A. Appl. Phys. Lett. 2001, 79, 872-874.

(15) Zhang, J.; Alsayed, A.; Lin, K. H.; Sanyal, S.; Zhang, F.; Pao, W. J.; Balagurusamy, V. S. K.; Heiney, P. A.; Yodh, A. G. Appl. Phys. Lett. 2002, 81, 3176-3178.

(16) Goldenberg, L. M.; Wagner, J.; Stumpe, J.; Paulke, B. R.; Gornitz, E. Langmuir 2002, 18, 3319-3323.

(17) Hoogenboom, J. P. Thesis, Utrecht University, Utrecht, The Netherlands, 2002; available at http://www.colloid.nl.

(18) Yin, Y. D.; Xia, Y. N. Adv. Mater. 2002, 14, 605-608.

(19) Park, S. H.; Qin, D.; Xia, Y. Adv. Mater. 1998, 10, 1028-1032.

(20) Yi, D. K.; Kim, M. J.; Kim, D. Y. Langmuir 2002, 18, 2019-2023.

NL034867H 